\title{
THE CONFORMAL RIGIDITY OF CERTAIN SUBDOMAINS ON A RIEMANN SURFACE
}

\author{
BY \\ H. L. ROYDEN
}

Let $V$ be a finite Riemann surface, i.e., one which is either compact or bounded by a finite number of boundary contours $\Gamma$. By a quadratic differential on $V$ is meant an expression $d \zeta^{2}$ which, relative to a local uniformizer $z$, has the form

$$
d \zeta^{2}=h(z) d z^{2},
$$

where $h(z)$ is an analytic function depending on the uniformizer so that $d \zeta^{2}$ is independent of the uniformizer. Thus in terms of another local uniformizer $w$

$$
d \zeta^{2}=h(z)\left(\frac{d z}{d w}\right)^{2} d w^{2} .
$$

The differential $d \zeta^{2}$ is said to have a pole of order $k$ at a point $p$ if $h$ has a pole of order $k$ there. The point $p$ is often referred to as a pole of $d \zeta^{2}$. If $d \zeta^{2}$ is non-negative on the contours $\Gamma$, then $d \zeta^{2}$ is called a quadratic differential of $V$, every quadratic differential on a compact surface being a quadratic differential of the surface. A curve along which $d \zeta^{2}$ is positive is called a trajectory [3] of $d \zeta^{2}$.

It is known [7] that if $V_{1}$ is a subregion of $V$ with the property that there are no mappings of $V_{1}$ into $V$ which are arbitrarily close to the identity mapping of $V_{1}$ into $V$, then $V_{1}$ must be a dense subregion of $V$ whose boundary relative to $V$ consists of slits along which some quadratic differential $d \zeta^{2}$ of $V$ is non-negative. The principal result of this paper is the proof of a strong form of the converse of this fact, a proof that under the proper conditions there are not only no conformal mappings near the identity but no conformal mappings which are homotopic to the identity. More precisely we have the following theorem.

THEOREM. Let d $\zeta^{2}$ be a quadratic differential of a finite Riemann surface $V$, and let $V_{1}$ be a dense subregion of $V$ bounded by a finite number of arcs along which $d \zeta^{2}$ is non-negative and containing in its interior all the multiple poles of $d \zeta^{2}$. Then the identity is the only conformal mapping $f$ of $V_{1}$ into $V$ which has all of the following properties:

(i) there is an interior point of $V_{1}$ which is a fixed point of $f$;

(ii) the poles of $d \zeta^{2}$ which lie in $V_{1}$ are fixed points of $f$, and near a pole of

Received by the editors November 4, 1952. 
order $k \geqq 2$ the mapping has the form

$$
f(z)=z+a z^{k}+\cdots
$$

in terms of a uniformizer $z$ at the pole;

(iii) on $V_{1}^{\prime}$ there is a deformation in $V^{\prime}$ of $f$ into the identity mapping of $V_{1}^{\prime}$ into $V^{\prime}$, where the primes indicate surfaces formed by removing the points at which $d \zeta^{2}$ has poles.

The special case in which $V$ is a sphere and $d \zeta^{2}$ has a pole at a single point arises in the coefficient problem for schlicht functions. It is a consequence of the Teichmüller lemma [9], and shows the uniqueness of the extremal functions. The special case in which all poles are simple arises in the interpolation problem for schlicht functions [5] and again serves to prove the uniqueness of the extremal functions. In the interpolation problem this uniqueness has important consequences.

Condition (i) of the theorem is redundant where there are poles in the interior of $V_{2}$. However, from the case in which $V$ is a torus and $V_{1}$ a region on it bounded by a piece of a trajectory of one of the Abelian differentials of $V$, this condition is seen to be necessary in the absence of poles. A more complicated example shows that the fixed point requirement cannot be omitted for surfaces of Euler characteristic other than zero either, although the pairs $V$ and $V_{1}$ for which the theorem fails without it are in a sense degenerate.

The proof of the theorem is a modification of the length area principle of Grötzsch [2], but is extremely simplified by the use of the uniqueness of geodesics on saddle surfaces.

The theorem is proved by a series of lemmas. We introduce a metric by defining the length of a curve $\alpha$ to be

$$
\int_{\alpha}|d \zeta|,
$$

and the distance between two points to be the greatest lower bound of the lengths of all curves joining them. A spheroid of radius $\epsilon$ about a point $p$ is the set of all points whose distance from $p$ is less than $\epsilon$.

LeMma 1. We may choose the deformation $\phi(p, t)$ of $f$ to the identity so that the lengths of the curves $\{\phi(z, t): 0 \leqq t \leqq 1\}$ are $O\left(|z|^{k / 2}\right)$ uniformly in terms of a fixed uniformizer $z$ at a kth order pole of $d \zeta^{2}$ which lies in the interior of $V_{1}$.

Proof. Let $p^{*}$ be such a pole and let

$$
\psi: V_{1}^{\prime} \times I \rightarrow V^{\prime}
$$

be the given deformation of $f$, and let $W^{\prime}$ be the universal covering surface of $V^{\prime \prime}$, the surface formed by deleting from $V$ all poles of $d \zeta^{2}$ other than $p^{*}$. Taking a point $p^{\prime} \in W^{\prime}$ which covers $p^{*}$ we form the universal covering surface 
$\tilde{W}$ of $W^{\prime}$ punctured at all points lying over $p^{*}$ except $p^{\prime}$. Let $w$ be a uniformizer mapping $\tilde{W}$ onto the unit circle $|w|<1$ so that the point $w=0$ lies over $p^{*}$.

If $U$ is a simply connected neighborhood of $p^{*}$, there is a unique conformal mapping $g$ of $U$ onto a region $\tilde{U}$ of $\tilde{W}$ such that $g\left(p^{*}\right)=0$ and the projection of $g\left(p^{*}\right)$ is $p$. Upon a little reflection it can be seen that $\psi$ defines a mapping

$$
\tilde{\psi}: \tilde{U} \times I \rightarrow \tilde{W}
$$

with the property that the projection to $V^{\prime \prime}$ of $\tilde{\psi} \circ g$ is $\psi$. Similarly the mapping $f$ defines a conformal mapping $\tilde{f}$ of $\tilde{U}$ into $\tilde{W}$.

Choosing $r$ so small that the circle $K:\{|w| \leqq r\}$ lies in $U$ and taking $r^{\prime}<r$, we define a mapping

$$
\tilde{\phi}: K \times I \rightarrow \tilde{W}
$$

as follows:

$$
\tilde{\phi}(w, t)=t w+(1-t) \tilde{f}(w)
$$

for $|w|<r_{1}$, and

$$
\tilde{\phi}(w, t)=\frac{r-|w|}{r-r^{\prime}}[t w+(1-t) \tilde{f}(w)]+\frac{|w|-r^{\prime}}{r-r^{\prime}} \tilde{\psi}(w, t)
$$

for $r^{\prime} \leqq|w| \leqq r$. Clearly $\tilde{\phi}$ is continuous in $K \times I$ and agrees with $\tilde{\psi}$ on the boundary of $K \times I$. Thus the function defined on $V^{\prime \prime} \times I$ as the projection of $\tilde{\phi} \circ g$ in the projection of $K \times I$ and as $\psi$ elsewhere is continuous. Since $f$ has the form

$$
f(w)=w+a^{\prime} w^{k}+\cdots,
$$

and $d \zeta^{2}$ has the form

$$
\left(\frac{c}{w^{k}}+\cdots\right) d w^{2}
$$

the $d \zeta^{2}$-length of the curve $\{\tilde{\phi}(w, t): 0 \leqq t \leqq 1\}$ is uniformly $O\left(|w|^{k / 2}\right)$. The curve $\{\phi(z, t): 0 \leqq t \leqq 1\}$ is the projection of this and has the same length. Since projection is a conformal mapping, this length is uniformly $O\left(|z|^{k / 2}\right)$.

Repeating this process for the other poles of $d \zeta^{2}$ completes the proof of the lemma.

Lemma 2. Without loss of generality we may assume that $d \zeta^{2}$ has no simple poles.

Proof. Lemma 1 shows the deformation $\phi(p, t)$ to have the property that as $p$ approaches a simple pole $p^{*}$ in the interior of $V_{1}$ we have $\phi(p, t) \rightarrow p^{*}$. 
Let $\tilde{V}$ be a two-sheeted covering surface $\left.{ }^{1}\right)$ of $V$ which has simple branch points at the simple poles of $d \zeta^{2}$. On $\tilde{V}, d \zeta^{2}$ becomes a differential without simple poles, and there is a dense subregion $\tilde{V}_{1}$ on $\tilde{V}$ bounded by pieces of trajectories of $d \zeta^{2}$. The mapping $f$ defines a conformal mapping $\tilde{f}$ of $\tilde{V}_{1}$ into $\tilde{V}$ satisfying the hypotheses of Theorem 1 , since $\phi(p, t)$ defines a deformation of $\tilde{\phi}$ to the identity in $\tilde{V}$ punctured at the multiple poles of $d \zeta^{2}$. But the conclusion of Theorem 1 for $\tilde{f}$ implies the same conclusion for $f$.

Thus for the remainder of the proof we assume that $d \zeta^{2}$ has no simple poles. We say that two arcs are homotopic if they have the same beginning and end points and are homotopic in $V^{\prime}$.

It is a routine matter to give a triangulation of $V^{\prime}$ such that the function

$$
\zeta=\int\left(d \zeta^{2}\right)^{1 / 2}
$$

maps each triangle into a unique (except for translation and reflection) Euclidean triangle in the $\zeta$-plane. Moreover, the sum of the angles at a vertex of the triangulation is not less than $2 \pi$. By a geodesic is meant a curve $\gamma$ which is a straight line in each triangle and which has the property that at any point of $\gamma$ the angle sum on each side of $\gamma$ is not less than $\pi$. If a point of $\gamma$ is a boundary point of $V$, the angle sum at that point is defined on only one side of $\gamma$, but $\gamma$ is still called geodesic if the angle sum on that side is not less than $\pi$.

LEMma 3. In each homotopy class of curves joining two points together there is a unique geodesic, and it has shorter length than any other curve in the homotopy class. Also there is a unique geodesic in each nontrivial homotopy class of curves joining a point with itself.

Proof. Let $p$ and $q$ be the points, and let $\tilde{p}$ and $\tilde{q}$ be points lying over them on the universal covering surface of $V^{\prime}$ and such that an arc joining $\tilde{p}$ and $\tilde{q}$ projects into an arc belonging to the proper homotopy class. There are only a finite number of triangles of the covering surface which contain points closer to $\tilde{p}$ than $\tilde{q}$ is. Hence there is a simply-connected region $\Omega$ on the covering surface containing only a finite number of triangles and containing all points as close to $\tilde{p}$ as $\tilde{q}$ is. Since $\Omega$ is now a simply-connected saddle polyhedron, there is $\left(^{2}\right)$ by Theorem 1 of [8] a unique geodesic (of $\Omega$ ) joining $\tilde{p}$ and $\tilde{q}$, and it is shorter than any other arc joining $\tilde{p}$ with $\tilde{q}$. Since the boundary of $\Omega$ relative to the universal covering surface of $V^{\prime}$ is farther from $\tilde{p}$ than the length of this geodesic, this geodesic does not meet the boundary of $\Omega$ and thus projects into a geodesic on $V^{\prime}$. Similarly for nontrivial homotopy classes

(1) If $V$ is compact and $d \zeta^{2}$ has an odd number of simple poles, then we may first take an unbranched two-sheeted covering surface of $V$ and let $\widetilde{V}$ be a two-sheeted covering surface of that.

(2) The method used in [8] was already invented by Teichmüller in [9]. 
of arcs joining a point with itself.

Since every trajectory is a geodesic, it must have shorter length than any path homotopic to it. Hence we have the following lemma.

LEMma 4. Let $\alpha$ be a piece of a trajectory of $d \zeta^{2}$. Then for any arc $\beta$ homotopic to $\alpha$ we have

$$
\int_{\beta}|d \zeta| \geqq \int_{\alpha}|d \zeta|
$$

with equality only if $\beta$ is $\alpha$.

LEMMA 5. If $K$ is a compact subset of the interior of $V_{1}$, then there is a constant $l_{0}=l_{0}(K)$ such that for each $p \in K$ there is an arc $\gamma_{p}$ of length less than $l_{0}$ and with the property that the arc $\gamma_{p}+f(\alpha)-\gamma_{q}$ is homotopic to $\alpha$ for any arc $\alpha$ lying in $V_{1}$ and having end points $p, q \in K$.

Proof. Let $\theta_{p}$ be the curve $\{\phi(p, t): 0 \leqq t \leqq 1\}$. Then $\theta_{p}+f(\alpha)-\theta_{q}$ is homotopic to $\alpha$. Let $\gamma_{p}$ be the geodesic homotopic to $\theta_{p}$. Then $\gamma_{p}+f(\alpha)-\gamma_{q}$ is homotopic to $\alpha$, and it suffices to show that the length $l(p)$ of $\gamma_{p}$ is bounded for $p \in K$. Lemma 1 shows that $l$ is bounded near the poles of $d \zeta^{2}$. Thus we need only show that $l$ is continuous in $K \cap V^{\prime}$.

Given $p \in K \cap V^{\prime}$, let $U$ be a spheroid about $f(p)$ whose diameter is less than $\epsilon$ and so small that $U$ is simply-connected, and let $U_{1}$ be a spheroid about $p$ whose diameter is less than $\epsilon$ and so small that $f\left(U_{1}\right) \subset U$. For $q \in U_{1}$ the geodesics $\eta$ and $\eta^{*}$ which join $q$ with $p$ and $f(q)$ with $f(p)$, respectively, lie in $U_{1}$ and $U_{2}$, respectively, and have lengths less than $\epsilon$. Since $U_{1}$ is simply connected, $\eta^{*}$ is homotopic to $f(\eta)$, and we have

$$
\gamma_{p}-\eta^{*}-\gamma_{q}+\eta
$$

homotopic to zero. By the minimal length property of $\gamma_{p}$ and $\gamma_{q}$ we have

$$
|l(p)-l(q)|<2 \epsilon
$$

Thus $l(p)$ is continuous and the lemma follows.

Lemma 6. Let $M$ be a measure space with finite measure, $E$ a measurable subset of $M$, and $T$ a measure preserving transformation of $M$ onto itself. Then for almost all points $x \in E, T^{n} x$ belongs to $E$ for arbitrarily large positive and negative values of $n$.

Proof. Without loss of generality we may assume that $T$ is metrically transitive, for otherwise $M$ decomposes into a countable number of subspaces on each of which $T$ is metrically transitive. We may also assume $m E$ $>0$. With $T$ metrically transitive the lemma is a simple consequence of the ergodic theorem (cf. [4, p. 49]). For, letting $\chi(x)$ be the characteristic function of $E$, we see by the ergodic theorem that for almost all $x \in E$ 


$$
\lim \frac{1}{N} \sum_{n=0}^{N} \chi\left(T^{n} x\right)=\frac{m(E)}{m(M)}>0,
$$

which would be impossible were $T^{n} x$ to belong to $E$ for only a finite number of $n$. Similarly for negative values of $n$.

Choosing a fixed set of uniformizers, one for each pole of $d \zeta^{2}$, we denote by $V_{\epsilon}$ the region obtained by deleting a circle of radius $\epsilon$ about each pole of $d \zeta^{2}$ relative to the fixed uniformizer there.

Considering $f$ as a mapping from the $\zeta$-plane to the $\zeta$-plane, we use $f^{\prime}$ to designate its derivative. Thus $f^{\prime}$ is uniquely defined except for sign at each point of $V_{1}$, and for any region $\Omega \subset V_{1}$

$$
\iint_{f(\Omega)}|d \zeta|^{2}=\iint_{\Omega}\left|f^{\prime}\right|^{2}|d \zeta|^{2},
$$

while for any arc $\alpha$ lying in $V_{1}$

$$
\int_{f(\alpha)}|d \zeta|=\int_{\alpha}\left|f^{\prime}\right||d \zeta| .
$$

We define a measure space $M$ the elements of which are directed horizontal line segments lying in some one of the triangles of $V_{1}$ which meet $V_{e}$. We require the end points of one of these line segments to be either on an edge of the triangle containing it or else on the boundary $B$ of $V_{\mathrm{e}}$ relative to $V^{\prime}$. If $E$ is a subset of $M$ lying in a single triangle, we define $\nu_{E}(\eta)$ to be the number of elements of $E$ which project into $\eta$ (counting, of course, a line segment directed from right to left as distinct from the same segment directed from left to right). The function $\nu_{E}(\eta)$ is bounded since both $B$ and the sides of the triangle are analytic arcs on $V$. We call $E$ measurable if $\nu_{E}$ is and define the measure of $E$ by

$$
m(E)=\int \nu_{E}(\eta) d \eta
$$

This measure extends to arbitrary subsets of $M$ by additivity.

Lemma 7. We have

$$
\iint_{V_{\epsilon}}\left|f^{\prime}\right||d \zeta|^{2} \geqq \iint_{V_{\mathbf{c}}}|d \zeta|^{2}+O(\epsilon) .
$$

Proof. Each element of $M$ is a piece of a directed trajectory of $d \zeta^{2}$. As we prolong the trajectory in the given direction we either come to a zero of $d \zeta^{2}$, come to the boundary of $V_{1}$, come to $B$, or are able to prolong the trajectory indefinitely. This leads us to partition the elements of $M$ into the five subsets $M_{0}, M_{1}, M_{2}, M_{3}, M_{4}$ accordingly as the element is part of a trajectory 
which passes through a zero of $d \zeta^{2}$ or comes to the boundary of $V_{1}$, which begins and ends on $B$, which begins on $B$ but has no end, which ends on $B$ but has no beginning, or which has neither beginning nor ending. Since $M_{0}$ is countable, it has measure zero.

On $M$ we define two non-negative measurable functions

$$
g(x)=\int_{x}|d \zeta|
$$

and

$$
h(x)=\int_{x}\left|f^{\prime}\right||d \zeta|
$$

Now

$$
\iint_{V_{\epsilon}}|d \zeta|^{2}=\frac{1}{2} \int_{M} g(x)
$$

and

$$
\iint_{V_{0}}\left|f^{\prime}\right||d \zeta|^{2}=\frac{1}{2} \int_{M} h(x) .
$$

Thus proving the lemma is equivalent to proving

$$
\int_{M} h(x) \geqq \int_{M} g(x)+O(\epsilon) .
$$

Letting $M^{\prime}$ consist of those elements of $M$ which neither belong to $M_{0}$ nor end on $B$, we define a one-to-one transformation

$$
T: M^{\prime} \rightarrow M
$$

which takes an element $x$ of $M^{\prime}$ into that element of $M$ which follows $x$ on the directed trajectory of which $x$ is a part. Since $T$ is piecewise linear on a natural representation of $M$, it is measure preserving.

The set $M_{1}$ is the union of the disjoint subsets $M\left(p^{*}, q^{*}\right)$ consisting of those $x$ lying on trajectories beginning on $K_{p^{*}}$, the circle of $B$ enclosing the pole $p^{*}$, and ending on $K_{q^{*}}$, the circle enclosing the pole $q^{*}$ (not necessarily distinct from $p^{*}$ ). Assume that $p^{*}$ is a pole of order $k$ and $q^{*}$ a pole of order not less than $k$. Let $E$ be the set of those $x \in M\left(p^{*}, q^{*}\right)$ which begin on the circle $K_{p}$. Then

$$
m(E) \leqq \int_{K_{p}^{*}}|\operatorname{Im} d \zeta| \leqq \int_{K_{p}^{*}}|d \zeta|=O\left(\epsilon^{1-k / 2}\right) .
$$

Let $E_{0}$ consist of those $x \in E$ which end on $K_{q^{*}}$. The transformation $T$ is defined on $E-E_{0}$, and for each $x \in E-E_{0}$ there is some positive integer $n$ 
such that $T^{n} x$ ends on $K_{q^{*}}$. Denote the set of $x$ having this property for a given $n$ by $E_{n}$. The $E_{n}$ are all measurable, being in fact countable unions of intervals in a natural representation of $M$. For $x \in E_{n}$, the segments

$$
x+T x+\cdots+T^{n} x
$$

form a geodesic $\alpha$ beginning on $K_{p^{*}}$ and ending on $K_{q^{*}}$. Its length is

$$
g(x)+g(T x)+\cdots+g\left(T^{n} x\right),
$$

while the length of $f(\alpha)$ is $h(x)+h(T x)+\cdots+h\left(T^{n} x\right)$. If $p$ and $q$ are the end points of $\alpha$, then by Lemma 1 the curves $\theta_{p}=\{\phi(p, t): 0 \leqq t \leqq 1\}$ and $\theta_{q}$ $=\{\phi(q, t): 0 \leqq t \leqq 1\}$ have lengths which are uniformly $O\left(\epsilon^{k / 2}\right)$ and have the property that $\theta_{p}+f(\alpha)-\theta_{q}$ is homotopic to $\alpha$. Thus by Lemma 4

$$
l[f(\alpha)] \geqq l[\alpha]+O\left(\epsilon^{k / 2}\right),
$$

where $l[$ ] denotes "length of." Thus

$$
\sum_{i=0}^{n} g\left(T^{i} x\right) \leqq \sum_{i=0}^{n} h\left(T^{i} x\right)+O\left(\epsilon^{k / 2}\right)
$$

uniformly in $x$. Integrating over $E_{n}$ and remembering that $T$ is measure preserving,

$$
\int_{\bigcup_{T^{i} E_{n}}} g(x) \leqq \int_{\bigcup_{T^{i} E_{n}}} h(x)+m\left(E_{n}\right) O\left(\epsilon^{k / 2}\right)
$$

uniformly in $n$. Since

$$
M\left(p^{*}, q^{*}\right)=\bigcup_{n=0}^{\infty} \bigcup_{i=0}^{n} T^{i} E_{n}
$$

we have

$$
\int_{M\left(p^{*}, q^{*}\right)} g(x) \leqq \int_{M\left(p^{*}, q^{*}\right)} h(x)+m(E) O\left(\epsilon^{k / 2}\right)
$$

or

$$
\int_{M\left(p^{*}, q^{*}\right)} g(x) \leqq \int_{M\left(p^{*}, q^{*}\right)} h(x)+O(\epsilon) .
$$

We dispose similarly of the case in which the order of $q^{*}$ is less than that of $p^{*}$. Adding over all possible combinations of poles, we have

$$
\int_{M_{1}} g(x) \leqq \int_{M_{1}} h(x)+O(\epsilon) .
$$


The transformation $T$ is a measure preserving mapping of the set $M_{2}$ into itself, and so by the ergodic theorem the averages

$$
\bar{g}(x)=\lim _{N \rightarrow \infty} \frac{1}{N+1} \sum_{n=0}^{N} g\left(T^{n} x\right)
$$

and

$$
\bar{h}(x)=\lim _{N \rightarrow \infty} \frac{1}{N+1} \sum_{n=0}^{N} h\left(T^{n} x\right)
$$

exist for almost all $x \in M_{2}$.

Let $K$ be a finite collection of (compact) arcs lying in the interior of $V_{e}$ and having the property that the length of the projection on the $\eta$-axis of those $K$ lying in a triangle differs from the length of the projection of the triangle by less than $\delta$ divided by the number of triangles meeting $V_{\text {c. }}$ Let $E$ be the set of $x \in M_{2}$ which intersect $K$. Then the measure of $M_{2}-E$ is less than $2 \delta$. By Lemma 6 there are, for almost all $x \in E$, arbitrarily large values of $N$ for which $T^{N} x \in E$. Let $\tau$ be a piece of trajectory which begins at an intersection of $x$ with $K$ and ends with an intersection of $T^{N} x$ with $K$. Then

$$
l[\tau] \geqq \sum_{n=1}^{N-1} g\left(T^{n} x\right)
$$

Also

$$
l[f(\tau)] \leqq \sum_{n=0}^{N} h\left(T^{n} x\right)
$$

From Lemmas 4 and 5

$$
l[\tau] \leqq l[f(\tau)]+2 l_{0}
$$

where $l_{0}$ is the constant of Lemma 5 and depends only on $K$, not on $N$. Thus

$$
\sum_{n=1}^{N-1} g\left(T^{n} x\right) \leqq \sum_{n=0}^{N} h\left(T^{n} x\right)+2 l_{0},
$$

whence

$$
\bar{g}(x) \leqq \bar{h}(x)
$$

for almost all $x \in E$, and, since $\delta$ was arbitrary, for almost all $x \in M_{2}$. By the ergodic theorem

$$
\int_{M_{2}} g(x)=\int_{M_{2}} \bar{g}(x) \text { and } \int_{M_{2}} h(x)=\int_{M_{2}} \bar{h}(x) .
$$

Hence 


$$
\int_{M_{2}} g(x) \leqq \int_{M_{2}} h(x)
$$

Similarly for $M_{3}$ and $M_{4}$, proving the lemma, since $M_{0}$ has measure zero and contributes nothing.

LEMMA 8. If $\left|f^{\prime}\right|$ is not constant on $V_{1}$, there is a constant $\delta>0$ such that for all sufficiently small $\epsilon$

$$
\iint_{f\left(V_{\epsilon}\right)}|d \zeta|^{2}>\iint_{V_{\epsilon}}|d \zeta|^{2}+\delta
$$

Proof. If $\left|f^{\prime}\right|$ is not constant, then there are regions $\Omega_{1}$ and $\Omega_{2}$ both of measure greater than some positive $h$ on which $\left|f^{\prime}\right|>M$ and $\left|f^{\prime}\right|<m$, respectively, with $M>m$. If $\epsilon$ is so small that $V_{\epsilon}$ contains $\Omega_{1}$ and $\Omega_{2}$, then by an elementary sharpening of the Schwarz inequality

$$
\begin{aligned}
& \iint\left|f^{\prime}\right|^{2}|d \zeta|^{2} \cdot \iint|d \zeta|^{2} \\
& \geqq \frac{h}{2}(M-m)^{2} \iint|d \zeta|^{2}+\left\{\iint\left|f^{\prime}\right||d \zeta|^{2}\right\}^{2},
\end{aligned}
$$

all integrals being over $V_{\text {c. }}$ By Lemma 7

$$
\iint\left|f^{\prime}\right||d \zeta|^{2} \geqq \iint|d \zeta|^{2}+O(\epsilon),
$$

whence

$$
\left\{\iint\left|f^{\prime}\right||d \zeta|^{2}\right\}^{2} \geqq\left\{\iint|d \zeta|^{2}\right\}^{2}+\left\{\iint|d \zeta|^{2}\right\} \cdot O(\epsilon) .
$$

Thus

$$
\iint\left|f^{\prime}\right|^{2}|d \zeta|^{2} \geqq \iint|d \zeta|^{2}+\frac{h}{2}(M-m)^{2}+O(\epsilon) .
$$

Since

$$
\iint_{V_{e}}\left|f^{\prime}\right|^{2}|d \zeta|^{2}=\iint_{f\left(V_{\epsilon}\right)}|d \zeta|^{2}
$$

we have

$$
\iint_{f\left(V_{\epsilon}\right)}|d \zeta|^{2} \geqq \iint_{V_{\epsilon}}|d \zeta|^{2}+\delta
$$

for all sufficiently small $\epsilon$, proving the lemma. 
Lemma 9. We have $\left|f^{\prime}\right|$ identically constant on $V_{1}$.

Proof. Near a pole $p^{*}$ of order $k$ the symmetric difference of $V_{\boldsymbol{\epsilon}}$ and $f\left(V_{\boldsymbol{\epsilon}}\right)$ is contained in an annulus of radius $O(\epsilon)$ and width $O\left(\epsilon^{k}\right)$ in the $z$-plane. Thus the total $\zeta$-area of this symmetric difference is $O(\epsilon)$ since $d \zeta^{2}=d z^{2} O\left(\epsilon^{-k}\right)$. Thus

$$
\iint_{V_{\epsilon}}|d \zeta|^{2}=\iint_{f\left(V_{\epsilon}\right)}|d \zeta|^{2}+O(\epsilon) .
$$

This and Lemma 8 imply the present lemma.

Leмma 10. The mapping $f$ is the identity.

Proof. If $d \zeta^{2}$ has a multiple pole, $f^{\prime}$ must be identically one near the pole since it is constant in a neighborhood of the pole $\left(\left|f^{\prime}\right|\right.$ being constant there) and approaches one as we approach the pole. Thus $f$ is the identity near the pole and must be the identity everywhere on $V_{1}$ by analytic continuation.

Suppose, on the other hand, that $d \zeta^{2}$ is everywhere regular. Then in the neighborhood of the fixed point $p$ of $f$ we have

$$
\left|f^{\prime}(z)\right|=\text { const. }
$$

or

$$
f(z)=c z
$$

where $z$ is that uniformizer at $p$ which is of the form

$$
z=[\zeta-\zeta(p)]^{\alpha} \text {. }
$$

Since $f$ is a linear mapping (in terms of $\zeta$ ) in the neighborhood of each point, it takes geodesics into geodesics. Since $d \zeta^{2}$ is regular, $V_{1}$ cannot be simply connected, and so there is by Lemma 3 a nontrivial geodesic $\gamma$ joining $p$ with itself. Since $\gamma$ is unique in its homotopy class, $f(\gamma)$ must be $\gamma$, and consequently $\gamma$ and $f(\gamma)$ have the same length, and thus the constant value of $\left|f^{\prime}\right|$ is one. There must now be a point $q$ in the interior of $\gamma$ for which $f(q)=q$. Let $\gamma^{\prime}$ be a geodesic from $p$ to $q$. Since $f\left(\gamma^{\prime}\right)=\gamma^{\prime}$, the direction of $\gamma^{\prime}$ at $p$ is preserved by $f$. Thus

$$
f(z)=z
$$

near $p$, and so $f$ is the identity everywhere on $V_{1}$ by analytic continuation.

\section{BibliograPhy}

1. L. Ahlfors and A. Beurling, Invariants conformes et problèmes extrémaux, Dixième Congrès des Mathématiciens Scandinaves, 1946.

2. H. Grötzsch, Über die Verzerrung bei schlichter konformer Abbildung mehrfach-zusammenhängender schlichter Bereiche, Berichte über die Verhandlung der Sächsichen Akademie der Wissenschaften zu Leipzig. Mathematisch-physiche Klasse vol. 83 (1931) pp. 283-297. 
3. J. A. Jenkins and D. C. Spencer, Hyperelliptic trajectories, Ann. of Math. vol. 53 (1951) pp. 4-35.

4. E. Hopf, Ergodentheorie, Berlin, 1937.

5. H. L. Royden, The interpolation problem for schlicht functions, to appear.

6. A. C. Schaeffer and D. C. Spencer, Coefficient regions for schlicht functions, Amer. Math. Soc. Colloquium Publications, vol. 35, 1950.

7. M. Schiffer and D. C. Spencer, Functionals of finite Riemann surfaces, Princeton, 1952.

8. M. Shiffman, On the isoperimetric inequality for saddle surfaces with singularities, Courant Anniversary Volume, New York, 1948, pp. 383-394.

9. O. Teichmüller, Ungleichungen zwischen den Koeffizienten schlichter Funktionen, Preuss. Akad. Wiss. Sitzungsber. (1938) pp. 363-375.

10. - Extremale quasikonforme Abbildungen und quadratische Differentiale, Abhandlungen Preussischen Akademie der Wissenschaften. Mathematisch-Naturwissenschaftliche Klasse (1940) pp. 1-197.

STANFord UNiversity,

Stanford, Calif. 